Valeur de marché et mesures comptables : les entreprises de la nouvelle économie française.

\author{
Octobre 2006 \\ Frédérique EVRAERT \\ CREFF \\ Université Montesquieu Bordeaux IV \\ IAE Bordeaux \\ Email : frederique.evraert@wanadoo.fr
}




\section{Valeur de marché et mesures comptables : les entreprises de la nouvelle économie française.}

Octobre 2006

\section{Résumé}

Cet article s'intéresse à l'évaluation des entreprises de la nouvelle économie française. Il examine le lien entre leur valeur de marché et les principaux paramètres de mesure de la situation financière décrits dans les états financiers: le résultat, les flux de trésorerie et les capitaux propres. Les exercices 2000-2002 servent de cadre temporel à l'analyse. Cette recherche s'inscrit dans la lignée des études visant à montrer la pertinence des mesures comptables et l'utilité de l'information financière pour expliquer les valorisations boursières des sociétés. La méthodologie utilisée est celle des études d'association. Les résultats testés à l'aide de trois modèles montrent l'intérêt du résultat de l'exercice et du résultat opérationnel dans l'explication des valeurs de marché des entreprises.

Mots clés : facteurs de valeur, start up, nouvelle économie, études d'association, contenu informationnel des flux de résultat et de trésorerie, cycle boursier 2000-2002.

\section{Market Value and Accounting Measures: Start Up Companies in France, 2000-2002.}

This paper conveys further evidence on the financial valuation of French start up companies over the period 2000-2002.Using association studies, relevant indicators as net income versus cash flows are provided for explanation of market value and as proxy's of growth opportunities. Results show the relevance of accounting models and numbers, particularly the ability of net income and operating income to convey useful information for financial analysts in different business settings showing the persistency of usefulness of fundamental analysis when data is available.

Key Words: Value drivers, start up companies, new economy, association studies, informational content of net income and cash flows, market cycle. 


\section{Introduction}

Cet article apporte de nouveaux développements concernant les facteurs d'évaluation des entreprises françaises. Il considère d'une part le cas des sociétés cotées de la nouvelle économie souvent réputées atypiques et particulièrement volatiles et d'autre part les exercices comptables 2000-2002 qui ont connu des évaluations excessives suivies d'une chute brutale des cours. Pour le type de firmes étudiées, l'absence d'historique, l'originalité et la nouveauté du modèle d'affaires, des secteurs, des produits et des services offerts au public ont pu constituer un défi pour les analystes. Dans ce contexte les études antérieures faites par Myers (1977), Barth, Beaver et Landsman (1996), Ohlson (1995) et Dechow (1994) présentent un intérêt particulier. La recherche utilise la méthodologie des études d'association et un échantillon de 96 sociétés cotées pour lesquelles les différentes variables caractéristiques du résultat, des flux de trésorerie et du patrimoine des entreprises sont successivement testées.

La première partie présente la revue de la littérature qui a permis d'élaborer les hypothèses de recherche. La deuxième partie traite de la méthodologie et présente les modèles retenus. La dernière partie est consacrée à la présentation et à l'analyse des résultats obtenus.

\section{$\underline{\text { 1. Revue de la littérature et hypothèses }}$}

L'explication de la différence entre la valeur boursière des entreprises et leur valeur comptable est une question de recherche récurrente en comptabilité financière. La valeur comptable dépend des fonds propres investis alors que la capitalisation boursière fluctue en permanence par les ordres d'achat et de vente sur le marché, phénomène accru pendant les périodes haussières. Assez récemment Fama et French (1995) se sont intéressés à l'écart entre ces deux valeurs à l'aide du ratio «market to book» en montrant l'existence d'un lien positif entre ce ratio et les rendements boursiers futurs. Plusieurs explications ont été proposées pour justifier cette relation (Cazavan-Jeny, 2004) : l'existence d'une prime de risque (Fama et French, 1995) ${ }^{1}$, le "mispricing" ou mauvaise évaluation systématique du prix des titres par les investisseurs 
(Frankel et Lee, 1998) et les erreurs de sélection affectant les échantillons utilisés (Kothari, Shanken et Sloan, 1995).

D'autres explications ont été données pour justifier cette association positive, notamment par Amir et Lev (1996) et Lev et Sougiannis (1996). Pour ces auteurs, le modèle comptable du coût historique se concentre sur le coût et non sur la valeur ce qui justifie la prise en compte d'autres éléments immatériels et/ou physiques valorisés par les investisseurs. Le ratio «market to book» comme les éléments inducteurs de valeur apparaissent comme des approximations du «capital immatériel» des firmes non reflété dans le bilan.

Plus généralement, l'article de 1996 d'Amir et Lev a examiné l'importance pour les investisseurs des informations financières et non financières dans le secteur de la téléphonie mobile. Les auteurs soulignent ainsi la complémentarité entre information financière et non financière et la pertinence d'indicateurs autres que le résultat net et les flux de trésorerie pour évaluer les entreprises.

En fait, les recherches pour expliquer les valorisations boursières à partir des données comptables paraissent s'organiser autour de ces deux voies, la recherche d'éléments comptables capables de justifier les valorisations obtenues et la recherche d'éléments non financiers en complément de l'information financière.

\subsection{L’influence des flux financiers : résultat et trésorerie}

Certains travaux essaient d'identifier les indicateurs comptables expliquant le mieux la valeur boursière des entreprises. Le résultat net et les flux de trésorerie sont les plus étudiés. Dechow (1994), examinant la relation entre les grandeurs comptables et la rentabilité boursière des titres, montre la supériorité des indicateurs de rentabilité. En pratique, les recherches sur les marchés financiers américains montrent que les bénéfices nets présentent un contenu informationnel plus élevé que les flux de trésorerie d'activité (Biddle, Seow et Siegel, 1995 ; Rayburn, 1986 ; Sloan, 1996) même si plusieurs études nuancent ces résultats en tenant compte du secteur d'activité 
$\left(\right.$ Biddle, Seow et Siegel, 1995) ${ }^{2}$ et du cycle de vie de l'entreprise (Anthony et Ramesh, 1992 ; Black, 1998) pour approfondir l'analyse.

Déjà en 1992, Anthony et Ramesh avaient montré que la réponse du marché des actions aux ventes et aux investissements non anticipés est fonction du cycle de vie, vérifiant ainsi le propos de Bernard (1989) ${ }^{3}$ liant la pertinence du type d'information comptable publiée aux étapes de la croissance des entreprises.

Dans la continuité des travaux d'Anthony et Ramesh (1992), Black (1998) s'est intéressée au contenu informationnel des indicateurs de flux de trésorerie et du bénéfice net pour six étapes du développement des entreprises, du stade du démarrage à celui du déclin. Les flux de trésorerie étaient ceux dont l'agrégat constitue la variation de la trésorerie globale des entreprises : flux d'activité, d'investissement et de financement.

Black $(1998)^{4}$ montra alors que les flux de trésorerie présentent un contenu informationnel supérieur au résultat net lorsque l'entreprise est en période de croissance et/ou de forte incertitude. Ainsi, pour les entreprises en démarrage, seul le flux de trésorerie utilisé pour l'investissement est significatif. La capacité d'investissement apparaît comme le facteur clé de la réussite. Pour les entreprises de croissance, les seules variables significatives sont les flux de trésorerie d'activité et d'investissement, les premières ayant le contenu informationnel le plus élevé. Pour les entreprises en déclin, les flux de trésorerie d'activité sont encore déterminants. Seules les entreprises en phase de maturité se distinguent, le résultat rejoignant les trois indicateurs de flux de trésorerie pour s'associer à la valeur de marché, en présentant même le contenu informationnel le plus élevé $\left(\mathrm{R}^{2}: 0.87\right.$ contre 0.82 pour l'excédent de trésorerie globale et 0.81 pour le flux de trésorerie utilisé par l'investissement).

Ces résultats montrent que les mesures comptables jouent un rôle d'explication des valeurs de marché et que, pour les « start up » et entreprises de croissance, les indicateurs de résultat et de flux de trésorerie naturellement liés, alternent comme indicateurs prédictifs de valeur, avec une préférence possible pour la trésorerie. La survie à long terme de ces entreprises dépend semble-t- 
il de leur capacité d'investissement et de financement. On sait qu'en période de démarrage et de croissance beaucoup d'entreprises échouent souvent par manque de fonds propres et de trésorerie. Les flux de trésorerie d'activité mesurent la capacité de survie et de croissance interne et ceux de financement l'aptitude à lever des fonds externes pour la croissance. Les flux relatifs à l'investissement indiquent la capacité d'acquérir des immobilisations en vue de saisir les opportunités de croissance. D’un autre coté, outre sa force probante rappelée par Beaver (1996), Dechow (1994), Biddle et al. (1995), Rayburn (1986) et Sloan (1996), le résultat net est ce qui reste théoriquement à l'entrepreneur une fois déduites toutes les charges et le résultat opérationnel est depuis longtemps utilisé par les grandes entreprises pour éprouver la performance de leurs divisions et filiales. Nous testerons donc les hypothèses suivantes :

H1 : Pour les entreprises de la nouvelle économie, les mesures comptables lorsqu'elles sont disponibles sont positivement associées à la capitalisation boursière, éclairant ainsi utilement le jugement des analystes.

H2: Pour les entreprises de la nouvelle économie, le résultat net présente un contenu informationnel plus important que les flux de trésorerie.

H3 : Parmi les flux de trésorerie, ceux provenant de l'activité dominent plutôt que ceux relatifs à l'investissement et au financement.

\subsection{Indicateurs complémentaires et opportunités de croissance}

Le deuxième courant de recherche, initié par Amir et Lev, a souligné l'importance des variables non financières pour expliquer les capitalisations boursières des entreprises opérant dans des secteurs innovants aux fortes opportunités de croissance. Ces auteurs ne contestent pas l'utilité des mesures financières traditionnelles telles que le résultat net et les flux de trésorerie comme 
facteurs explicatifs de la valeur de marché mais s'interrogent sur des indicateurs alternatifs pertinents pour l'évaluation de sociétés particulières telles que celles de la nouvelle économie dont les résultats et les flux de trésorerie, souvent négatifs, rendent l'appréhension de la création de valeur difficile.

Aux Etats-Unis, les travaux sur l'évaluation des entreprises internet reconnaissent que le modèle comptable informe peu sur les opportunités de croissance et sur les actifs immatériels qui sont une composante essentielle de la valeur de ces entreprises. Les résultats concernant le rôle informatif du résultat net sont contradictoires (Hand, 2003 ;Trueman, Wong et Zhang, 2001; Rajgopal, Kotha et al., 2000a et 2003). Mais les conclusions concordent lorsque le compte de résultat est éclaté en composantes. La prise en compte de la recherche et développement et des dépenses commerciales apporte un contenu informationnel complémentaire. Tout se passe comme si ces dépenses et le cas échéant les pertes supportées étaient perçues par le marché comme des investissements. Les mesures physiques caractéristiques du trafic sur internet jouent aussi un rôle. En revanche, les variables de trésorerie et les marges ont retrouvé leur importance décisive après l'effondrement du marché haussier américain au printemps 2000 (Demers et Lev, 2000), les dépenses redevenant appréciées comme des charges et non plus comme des investissements (Demers et Lev, 2000 ; Trueman, Wong et Zhang, 2001 et 2002).

Selon Myers (1977), les actifs spécifiques et les options de croissance sont positivement corrélés. Trois mesures sont souvent choisies pour saisir la spécificité des actifs des entreprises : les dépenses de R\&D, celles de marketing et la valeur ajoutée (Cavanaugh et Garen, 1997). Pour la plupart des sociétés de notre échantillon un tel niveau de détail de l'information n'est pas disponible, le compte de résultat français établi par nature n'isolant pas les dépenses de R\&D et de marketing, informations que les sociétés commencent à publier dans l'annexe des comptes (Ding et Stolowy, 2003). En l'absence d'une décomposition fine des emplois du compte du résultat et de la faible disponibilité des mesures de trafic sur la toile, on ne peut pour estimer et évaluer l'impact des opportunités de croissance qu'utiliser des indicateurs de niveau d'activité. 
Les hypothèses complémentaires choisies sont les suivantes :

H4 : Les opportunités de croissance estimées par le chiffre d'affaires, la valeur ajoutée et le résultat opérationnel apportent une information complémentaire pour expliquer la capitalisation boursière des entreprises de la nouvelle économie.

H5 : Parmi les indicateurs de résultat, l'excédent brut d'exploitation choisi comme mesure du résultat opérationnel offre le contenu informationnel le plus élevé.

\section{$\underline{\text { 2. Méthodologie de l'étude }}$}

On étudie les entreprises de la nouvelle économie, particulièrement celles liées au développement d'Internet et des marchés numériques. Trois modèles servent à sélectionner les indicateurs pertinents pour expliquer les capitalisations boursières et ceux au pouvoir informationnel le plus élevé.

\subsection{Echantillon, choix des variables et statistiques descriptives \\ - L'échantillon}

Il comprend 96 sociétés cotées françaises. Le critère de sélection est l'appartenance à l'indice ITCAC $^{5}$, indice technologique français et la cotation sur le nouveau marché, marché des valeurs de croissance françaises. L'échantillon comprend des entreprises de nouvelles technologies et des valeurs de croissance. La période étudiée s'étend du $1^{\text {er }}$ janvier 2000 au 31 décembre 2002, période particulièrement volatile sur l'ensemble des marchés financiers mondiaux.

Les données extraites de la base de données OSIRIS sont complétées par les rapports annuels des sociétés ${ }^{6}$. L'échantillon est ensuite restreint aux entreprises pour lesquelles les données, notamment celles concernant les flux de trésorerie sont disponibles. Au total 245 données observations sont utilisables, une fois éliminées les observations extrêmes. 


\section{- Choix et présentation des variables.}

Les variables (tableau 1) sont les flux de trésorerie, le résultat net, la capitalisation boursière en fin d'année comptable, les fonds propres et les différents indicateurs d'activité et de performance destinés à représenter les opportunités de croissance : chiffre d'affaires, valeur ajoutée, excédent brut d'exploitation.

Tableau 1 : Notations et Variables

\begin{tabular}{|l|l|}
\hline Notations & Variables \\
\hline CAPI & Capitalisation boursière ( valeur de marché des fonds propres) \\
\hline FTA & Flux de trésorerie d'activité \\
\hline FTI & Flux de trésorerie d'investissement \\
\hline FTF & Flux de trésorerie de financement \\
\hline FP & Valeur comptable des fonds propres \\
\hline RN & Résultat net \\
\hline CA & Chiffre d'affaires \\
\hline EBE & Excédent brut d'exploitation \\
\hline VA & Valeur ajoutée \\
\hline
\end{tabular}

\section{- Statistiques descriptives}

Elles montrent une grande diversité de taille, de croissance et de performance boursière. Les paramètres de dispersion des variables retenues dans l'analyse et de certaines variables caractéristiques sont présentés en annexe. L'actif médian des entreprises de nouvelles technologies françaises est de 30 millions d'euros, le chiffre d'affaires de 28 millions d'euros. Il existe une forte disparité entre les sociétés mais la plupart des grandeurs structurelles étudiées, le total de l'actif, les fonds propres, le chiffre d'affaires, les actifs et les dettes d'exploitation font preuve de stabilité. En revanche, la capitalisation boursière est pratiquement divisée par quatre en trois exercices, les pertes ne cessent d'augmenter et les indicateurs de flux de trésorerie font l'objet de variations sensibles. In fine, la structure des sociétés reflétée dans les états financiers, ne s'est que peu modifiée en trois ans à la grande différence de la capitalisation boursière.

\section{- Analyse des corrélations}


Tableau 2 : Matrice des corrélations de Pearson entre capitalisation boursière, résultat et flux de trésorerie.

\begin{tabular}{|l|r|r|r|r|r|}
\hline CAPI/2002 & FP2002 & FTA02 & FTI02 & FTF02 & RN02 \\
&, $510(* *)$ & $-0,001$ &,$- 485(* *)$ &, $576(* *)$ &, $149(*)$ \\
FP2002 & 0 & 0,99 & 0 & 0 & 0,018 \\
& &,$- 347(* *)$ &,$- 575(* *)$ &, $589(* *)$ &,$- 154(*)$ \\
FTA02 & & 0,000 & 0,000 & 0,000 & 0,015 \\
& & &, $127(*)$ &,$- 427(* *)$ &, $463(* *)$ \\
FTI02 & & 0,047 & 0,000 & 0,000 \\
& & &,$- 831(* *)$ &,$- 145(*)$ \\
FTF02 & & & 0,00 & 0,02 \\
& & & & 0,002 \\
** significatif au seuil de 1\%0.01 (bilatéral). * significatif au seuil de 5\%(bilatéral). & 0,976 \\
CAPI : capitalisation boursière; FP : les fonds propres comptables; FTA : les flux de trésorerie \\
d'activité; FTI : les flux de trésorerie d'investissement; FTT : les flux de trésorerie de financement. \\
\hline
\end{tabular}

L'analyse des corrélations entre les variables révèle que la capitalisation boursière est corrélée positivement au montant des fonds propres, négativement au flux de trésorerie d'investissement et positivement au flux de trésorerie de financement ainsi qu'au résultat net. Les corrélations entre variables explicatives indiquent une relation forte entre fonds propres et flux de trésorerie. Ceux d'investissement et de financement sont négativement corrélés.

L'analyse des corrélations entre les différents indicateurs de performance montre une très importante corrélation entre le chiffre d'affaires, le total des produits, le coût des biens et services vendus, le montant des dotations aux amortissements et provisions, et les autres charges d'exploitation; et une forte corrélation entre résultat d'exploitation et excédent brut d'exploitation.

Pour éviter la multi-colinéarité des variables explicatives, le chiffre d'affaires est choisi comme indicateur de niveau d'activité avec en complément la valeur ajoutée pour la croissance et l'excédent brut d'exploitation comme indicateur de performance d'exploitation.

\section{- Effets contingents}

Pour tenir compte de la spécificité des entreprises et pour le contrôle des résultats, l'analyse retient plusieurs éléments de contingence. Le pouvoir explicatif du modèle est testé selon le milieu externe, la performance, le «business model» et l'âge des entreprises. 
Le milieu externe, ici la conjoncture, devrait exercer quelque influence. L'analyse est faite sur trois exercices commençant le ler janvier 2000 et s'achevant le 31 décembre 2002. Les valorisations boursières n'ont pas varié de façon linéaire pendant la période. En 2000, le cycle est haussier, beaucoup de sociétés sont introduites en bourse. En 2001, la situation s'inverse, la capitalisation boursière chute et la situation s'aggrave encore en 2002. Cela affecte les comportements des agents et les décisions prises.

La performance des entreprises est la deuxième métrique testée. On se demande s'il existe des différences d'évaluation pour les entreprises bénéficiaires et déficitaires.

Par ailleurs, les entreprises étudiées sont originales et leurs produits peu connus. Leurs domaines d'activité sont nouveaux : les nouvelles technologies de l'information et des communications. Cet effet secteur ou business model est étudié en divisant la population en trois groupes d'entreprises ${ }^{7}:$ « internet », « technologies de l'information » et « autres valeurs de croissance ». La quatrième métrique est l'ancienneté ou âge de l'entreprise. Elle classe les firmes d'après leur cycle de vie, mesurant ainsi leur capacité à la continuité d'exploitation : introduites en bourse avant le $1^{\text {er }}$ janvier 2000 ou après. Les analyses de régression sont faites pour l'ensemble de la période et pour chaque année, les autres facteurs de contingence : performance, secteur et âge sont aussi testés.

\subsection{Les modèles utilisés}

Ils s'inspirent de la définition par Myers (1977) de la valeur de l'entreprise et des modèles proposés dans la même lignée par Burgstalher et Dichev (1997), Barth, Beaver et Landsman (1996) et du modèle d'Ohlson modifié (1995).

\subsubsection{La valeur de l'entreprise d'après Myers (1977)}

Pour Myers (1977), la valeur de l'entreprise est égale à la valeur des actifs en place majorée de la valeur des opportunités de croissance. 
Modèle 1 : Myers (1977)

Valeur de l'entreprise $=$ Valeur des actifs en place + Valeur des opportunités de croissance

Cette définition intègre la dynamique d'évolution de l'entreprise, la valeur s'adaptant aux phases de développement. Selon la place dans le cycle de vie, le poids des actifs et des opportunités de croissance change : par exemple, la valeur d'une entreprise naissante («start up ») dépend principalement de ses opportunités de croissance, alors que pour une entreprise en phase de maturité, elle s'appuie sur les actifs contrôlés.

\subsubsection{La valeur de l'entreprise selon Barth et Beaver et Landsman (1996)}

Barth, Beaver et Landsman (1996) proposent une définition de la valeur de l'entreprise proche de celle de Myers. La valeur de l'entreprise ou capitalisation boursière est égale à la somme des actifs reconnus mesurée par la valeur comptable des fonds propres majorée de la valeur des actifs non comptabilisés («Unrecognized Assets : UNA »).

Modèle 2 : Barth, Beaver, Landsman (1996)

MVEit $=\mathrm{a}_{1}$ BVEit $+\mathrm{a}_{2}$ UNAit

MVEit: Capitalisation boursière de l'entreprise i à la date t; BVEit : Fonds propres de l'entreprise i à la date $\mathrm{t}$; UNAit reflète les opportunités de croissance de l'entreprise ; elles sont approximées par CFO, CFI, CFF et $\mathrm{RN}^{8}$.

Les auteurs analysent la valeur de l'entreprise comme une fonction à la fois de la valeur des fonds propres et des éléments non pris en compte en comptabilité. La difficulté vient de l'estimation des opportunités de croissance non directement réfléchies par les mesures comptables.

Bernard (1994), Barth, Beaver et Landsman (1996) et Ohlson (1995) utilisent le résultat comptable comme estimateur des opportunités de croissance des entreprises. 


\subsubsection{La valeur de l'entreprise d'après Ohlson (1995)}

Le modèle d'Ohlson modifié, modèle du bénéfice net résiduel est l'un des plus utilisés en comptabilité financière. Pour certains, il constitue la seule formalisation théorique du lien entre les chiffres comptables et le prix des titres.

La valeur de l'entreprise y est égale à la somme des fonds propres comptables majorée de la valeur actuelle des rendements anormaux futurs.

\section{Modèle 3 : Ohlson (1995)}

$\mathrm{MVE}_{\mathrm{i}, \mathrm{t}}=\mathrm{BVE}_{\mathrm{i}, \mathrm{t}}+\frac{\mathrm{E}\left(\mathrm{RN}_{\mathrm{i}, \mathrm{t}+1}\right)}{\left(1+\mathrm{r}_{\mathrm{i}}\right)^{\mathrm{i}}}$

MVEit: Capitalisation boursière de l'entreprise i à la date t; BVEit : Fonds propres de l'entreprise i à la date $t$;

$\mathrm{E}(\mathrm{RNi}, \mathrm{t}+1) /(1+\mathrm{ri})^{\mathrm{i}}$ Bénéfice net résiduel et $\mathrm{r}$, taux d'actualisation approprié.

Les trois modèles soulignent l'importance de l'évaluation des opportunités de croissance. Ils correspondent aux trois régressions testées ci-après, pour apprécier les indicateurs capables de mieux estimer les « opportunités de croissance », «les actifs net non reconnus » ou « la valeur actuelle des résultats futurs anormaux » des entreprises de la nouvelle économie.

\section{Les résultats}

Les résultats des modèles 1 (Myers) et 2 (Barth, Beaver, Landsman) sont présentés successivement. Le premier modèle teste le contenu informationnel des indicateurs de résultat net et de flux de trésorerie. On cherche ensuite par le Chi deux de Wald $^{9}$ si les variables ont un contenu informationnel différent. Le modèle 2 sélectionne ensuite l'indicateur présentant le pouvoir informationnel le plus élevé. Le modèle 3 (Ohlson) cherche in fine à améliorer la compréhension de la valeur. 


\subsection{Les résultats du modèle de Myers et de Barth et al. pour l'ensemble de la période}

Pour tester les contenus informationnels des indicateurs de flux de trésorerie et du résultat net, la régression suivante est réalisée pour l'échantillon et la période 2000-2002 (245 données observations).

Equation de régression $1: \mathrm{CB}_{\mathrm{i}, \mathrm{t}}=\mathrm{a}_{0}+\mathrm{a}_{1} \mathrm{Fp}_{\mathrm{it}}+\mathrm{a}_{2} \mathrm{Rn}_{\mathrm{it}}+\mathrm{a}_{3} \mathrm{FT}_{\mathrm{it}}+\mathrm{e}_{\mathrm{it}}$.

Avec $\mathrm{CB}_{\text {it }}$ : Capitalisation boursière de l'entreprise i à l'instant $\mathrm{t}$;

$F p_{i t}$ : Valeur comptable des fonds propres de l'entreprise i à l'instant $\mathrm{t}$;

$\mathrm{Rn}_{\mathrm{it}}$ : Résultat net de l'entreprise i à l'instant $\mathrm{t}$;

$\mathrm{FT}_{\mathrm{it}}$ : Indicateur de flux de trésorerie de l'entreprise i à l'instant $\mathrm{t}$;

$\mathrm{e}_{\mathrm{it}}$ : Terme d'erreurs.

Le deuxième modèle identifie l'indicateur au pouvoir explicatif le plus élevé pour la

capitalisation boursière. On cherche l'indicateur le plus apte à exprimer les opportunités de

croissance des entreprises de la nouvelle économie.

Le modèle testé est :

Equation de régression 2: $\mathrm{CB}_{i t}=\mathrm{b}_{0}+\mathrm{b}_{1} \mathrm{Fp}_{\mathrm{it}}+\mathrm{b}_{2} \mathrm{Una}_{\mathrm{it}}+\mathrm{e}_{\mathrm{it}}$

$\mathrm{CB}_{\mathrm{i}, \mathrm{t}}$ : Capitalisation boursière de l'entreprise i à l'instant $\mathrm{t}$;

$\mathrm{Fp}_{\mathrm{it}}$ : Valeur comptable des fonds propres de l'entreprise i à l'instant $\mathrm{t}$;

Una $_{\text {it }}$ : les actifs non reconnus de l'entreprise i pour la période $t$, estimées successivement par RN, FTA, FTI et FTF

$\mathrm{e}_{\text {it }}$ : terme d'erreurs

Le tableau 3 donne les résultats des régressions 1 et 2 . 
Tableau 3: Importance relative des flux de résultat net et de trésorerie (période complète)

Equations de régression du modèle 1:

$\mathrm{CB}_{\mathrm{i}, \mathrm{t}}=\mathrm{a}_{0}+\mathrm{a}_{1} \mathrm{FP}_{\mathrm{i}, \mathrm{t}}+\mathrm{a}_{2} \mathrm{RN}_{\mathrm{i}, \mathrm{t}+} \mathrm{a}_{3} \mathrm{FTA}_{\mathrm{i}}+\varepsilon_{\mathrm{i}, \mathrm{t}}$ (équation 1a)

$\mathrm{CB}_{\mathrm{i}, \mathrm{t}}=\mathrm{a}_{0}+\mathrm{a}_{1} \mathrm{FP}_{\mathrm{i}, \mathrm{t}}+\mathrm{a}_{2} \mathrm{RN}_{\mathrm{i}, \mathrm{t}+} \mathrm{a}_{3} \mathrm{FTI}_{\mathrm{i}}+\varepsilon_{\mathrm{i}, \mathrm{t}} \quad$ (équation $1 \mathrm{~b}$ )

$\mathrm{CB}_{\mathrm{i}, \mathrm{t}}=\mathrm{a}_{0}+\mathrm{a}_{1} \mathrm{FP}_{\mathrm{i}, \mathrm{t}}+\mathrm{a}_{2} \mathrm{RN}_{\mathrm{i}, \mathrm{t}+} \mathrm{a}_{3} \mathrm{FTF}_{\mathrm{i}}+\varepsilon_{\mathrm{i}, \mathrm{t}}$ (équation 1c)

\begin{tabular}{|c|c|c|c|}
\hline Régression 1 & \multicolumn{3}{|c|}{ Estimation des coefficients des équations } \\
\hline observations $=245$ & 1a (FTA) & 1b (FTI) & 1c (FTF) \\
\hline $\mathrm{C}$ (constante) & 7149.18 & 11042.91 & 15497.63 \\
\hline $\mathrm{FP}$ & 3.406* & $3.115 *$ & $2.582 *$ \\
\hline RN & $1.898 *$ & $2.52 *$ & $2.513 *$ \\
\hline FTA & $1.780 *$ & & \\
\hline FTI & & -0.359 & \\
\hline FTF & & & $1.087 *$ \\
\hline $\mathrm{R}^{2}$ & $49.12 \%$ & $47.3 \%$ & $49.5 \%$ \\
\hline Fisher ( p-value) & 0.000 & 0.000 & 0.000 \\
\hline Durbin-Watson & 2.014 & 1.84 & 1.913 \\
\hline Wald chi-deux stat & 0.016 & $26.41^{*}$ & $6.415^{*}$ \\
\hline $\mathrm{P}$ value & 0.898 & 0.000 & 0.0113 \\
\hline \multicolumn{4}{|c|}{$*_{\text {coefficients significatifs au seuil de } 5 \%}$} \\
\hline \multicolumn{4}{|c|}{$\begin{array}{l}\text { FP : Fonds propres; } \\
\text { RN: Résultat net; FTA : Flux de trésorerie d'activité; FTI : Flux de trésorerie d'investissement; FTF: Flux de } \\
\text { trésorerie de financement. }\end{array}$} \\
\hline
\end{tabular}

Equations de régression du modèle 2: $\mathrm{CB}_{\mathrm{i}, \mathrm{t}}=\mathrm{a}_{0}+\mathrm{a}_{1} \mathrm{FP}_{\mathrm{i}, \mathrm{t}}+\mathrm{a}_{2} \mathrm{UNA}_{\mathrm{i}, \mathrm{t}+} \varepsilon_{\mathrm{i}, \mathrm{t}}$

$\mathrm{CB}_{\mathrm{i}, \mathrm{t}}=\mathrm{a}_{0}+\mathrm{a}_{1} \mathrm{FP}_{\mathrm{i}, \mathrm{t}}+\mathrm{a}_{2} \mathrm{RN}_{\mathrm{i}, \mathrm{t}+} \varepsilon_{\mathrm{i}, \mathrm{t}}$ (équation $2 \mathrm{a}$ )

$\mathrm{CB}_{\mathrm{i}, \mathrm{t}}=\mathrm{a}_{0}+\mathrm{a}_{1} \mathrm{FP}_{\mathrm{i}, \mathrm{t}}+\mathrm{a}_{2} \mathrm{FTA}_{\mathrm{i}, \mathrm{t}+} \varepsilon_{\mathrm{i}, \mathrm{t}}$ (équation 2b)

$\mathrm{CB}_{\mathrm{i}, \mathrm{t}}=\mathrm{a}_{0}+\mathrm{a}_{1} \mathrm{FP}_{\mathrm{i}, \mathrm{t}}+\mathrm{a}_{2} \mathrm{FTI}_{\mathrm{i}, \mathrm{t}+} \varepsilon_{\mathrm{i}, \mathrm{t}}$ (équation 2c)

$\mathrm{CB}_{\mathrm{i}, \mathrm{t}}=\mathrm{a}_{0}+\mathrm{a}_{1} \mathrm{FP}_{\mathrm{i}, \mathrm{t}}+\mathrm{a}_{2} \mathrm{FTF}_{\mathrm{i}, \mathrm{t}+} \varepsilon_{\mathrm{i}, \mathrm{t}}$ (équation $2 \mathrm{~d}$ )

\begin{tabular}{|c|c|c|c|c|c|c|c|}
\hline$\frac{\text { Variables }}{\mathrm{N}=245}$ & $\begin{array}{c}\text { Equation2a: } \\
\text { RN }\end{array}$ & \multicolumn{2}{|c|}{ Equation 2b: FTA } & \multicolumn{2}{|c|}{ Equation 2c: FTI } & \multicolumn{2}{|c|}{ Equation 2d: FTF } \\
\hline $\mathrm{C}$ & 10029.43 & \multirow{2}{*}{\multicolumn{2}{|c|}{$\begin{array}{l}-3027.57 \\
\mathbf{3 4 4 3} *\end{array}$}} & \multicolumn{2}{|c|}{-668.15} & \multicolumn{2}{|c|}{1771.81} \\
\hline $\mathrm{FP}$ & $3.25 *$ & & & \multicolumn{2}{|c|}{$2.78 *$} & \multirow{2}{*}{\multicolumn{2}{|c|}{$2.417 *$}} \\
\hline $\mathrm{RN}$ & 2.618* & & & & & & \\
\hline FTA & & \multicolumn{2}{|c|}{$2.806 *$} & \multirow{3}{*}{\multicolumn{2}{|c|}{$-0.99 *$}} & & \\
\hline FTI & & & & & & & \\
\hline FTF & & & & & & 1.2 & $14 *$ \\
\hline $\mathbf{R}^{2}$ & 47.17 & \multicolumn{2}{|c|}{46.34} & \multicolumn{2}{|c|}{41.0} & 1.214 & \\
\hline \multicolumn{2}{|c|}{ Test d'hypothèse } & $\mathrm{RN}>\mathrm{FTA}$ & FTA $>$ RN & $\mathrm{RN}>\mathrm{FTI}$ & FTI $>$ RN & $\mathrm{RN}>\mathrm{FTF}$ & $\mathrm{FTF}>\mathrm{RN}$ \\
\hline \multicolumn{2}{|c|}{ Résultat attendu } & oui & non & oui & non & oui & non \\
\hline \multicolumn{2}{|c|}{ Comparaison des $\mathbf{R}^{2}$} & \multicolumn{2}{|c|}{$\begin{array}{l}\text { RN }: \mathrm{R}^{2}=47.17 \\
\text { FTA }: \mathrm{R}^{2}=46.3\end{array}$} & \multicolumn{2}{|c|}{$\begin{array}{l}\mathrm{RN}: \mathrm{R}^{2}=47.17 \\
\text { FTI }: \mathrm{R}^{2}=41\end{array}$} & \multicolumn{2}{|c|}{$\begin{array}{l}\mathrm{RN}: \mathrm{R}^{2}=47.17 \\
\mathrm{FTF}: \mathrm{R}^{2}=42.9\end{array}$} \\
\hline
\end{tabular}


Pour la période entière 2000-2002, le résultat net est l'indicateur le plus pertinent.

Les deux familles d'indicateurs $(1 \mathrm{a}, 1 \mathrm{~b}, 1 \mathrm{c})$ indiquent que le résultat net et les flux de trésorerie expliquent en partie les capitalisations boursières. La qualité de l'ajustement des modèles est satisfaisante puisque les $\mathrm{R}^{2}$ sont proches de $47 \%$. Seul le flux de trésorerie d'investissement n'est pas significatif.

Le résultat net, les flux de trésorerie d'activité et de financement sont significatifs et positivement associés à la valeur de marché des sociétés françaises de la nouvelle économie.

La régression 1c indique que le résultat net et le flux de trésorerie de financement informent le mieux $\left(\mathrm{R}^{2}=49.5 \%\right)$. Le test de $\mathrm{Wald}^{10}$ montre l'utilité respective des deux informations ( $\mathrm{p}$ value $=0.000$ ) mais n'indique pas laquelle présente le contenu informationnel le plus élevé. Le modèle 2 permet de vérifier pour quelle variable "UNA", le pouvoir explicatif est le plus fort. Le résultat net présente le contenu informationnel le plus élevé pour expliquer la capitalisation boursière $\left(\mathrm{R}^{2}=47.1\right)$. Son contenu informationnel est supérieur au flux de trésorerie de financement $\left(\mathrm{R}^{2}=42.9 \%\right)$.

\subsection{Résultats du modèle de Myers, de Barth, Beaver, Landsman et métriques de contingence}

Les résultats obtenus pour les variables de contrôle sont synthétisés dans le tableau 4. Ils montrent que l'évaluation par le résultat est d'importance première.

L'analyse par année met en évidence l'importance du résultat pour l'ensemble de la période mais les flux de trésorerie jouent aussi un grand rôle. Le résultat net présente le contenu informationnel le plus important à la fois en 2000 et en 2001. Pour les flux de trésorerie, ce sont ceux d'investissement qui sont significatifs en 2000 et ceux de financement en 2001. En 2000, la capacité à investir est perçue comme un facteur clé de succès ce qui est conforme aux hypothèses. En 2001, les besoins en ressources deviennent primordiaux en raison de la forte 
chute des marchés. Les flux de financement informent sur la capacité d'emprunt, de remboursement et d'augmentation du capital des entreprises.

Tableau 4. Résultats de l'ensemble des régressions ( modèles 1 et 2)

\begin{tabular}{|c|c|c|c|c|c|c|c|c|c|c|}
\hline \multicolumn{6}{|c|}{ Résultats du modèle 1} & \multicolumn{5}{|c|}{ Résultat du modèle 2} \\
\hline & & & & & & RN & FTO & FTI & FTF & \\
\hline & $\mathrm{RN}$ & FTO & FTI & FTF & Commentaire modèle 1 & $\mathrm{R}^{2}$ & $\mathrm{R}^{2}$ & $\mathrm{R}^{2}$ & $\mathrm{R}^{2}$ & $\begin{array}{l}\text { Commentaire modèle } \\
2\end{array}$ \\
\hline $\begin{array}{l}\text { Echantillon } \\
\text { Total } \\
\mathrm{N}=245\end{array}$ & oui & oui & non & oui & $\begin{array}{l}\text { Seul le coefficient } \\
\text { attaché au flux de } \\
\text { trésorerie } \\
\text { d'investissement n'est } \\
\text { pas significatif }\end{array}$ & 47.7 & 46.3 & 41 & 42.9 & $\begin{array}{l}\text { Le résultat net } \\
\text { présente le contenu } \\
\text { informationnel le plus } \\
\text { élevé. }\end{array}$ \\
\hline Année 2000 & oui & non & oui & non & $\begin{array}{l}\text { Le résultat net et le flux } \\
\text { de trésorerie } \\
\text { d'investissement sont } \\
\text { significatifs }\end{array}$ & 71.7 & 67.3 & 66.2 & 65.1 & $\begin{array}{l}\text { Le résultat net } \\
\text { présente le contenu } \\
\text { informationnel le plus } \\
\text { élevé }\end{array}$ \\
\hline Année 2001 & oui & non & non & oui & $\begin{array}{l}\text { Le résultat net et le flux } \\
\text { de trésorerie de } \\
\text { financement sont } \\
\text { significatifs }\end{array}$ & 34 & 34.6 & 29 & 31.5 & $\begin{array}{l}\text { Le résultat net } \\
\text { présente le contenu } \\
\text { informationnel le plus } \\
\text { élevé }\end{array}$ \\
\hline Année 2002 & $\mathrm{~ns}$ & ns & $\mathrm{ns}$ & $\mathrm{ns}$ & $\begin{array}{l}\text { Le modèle n'est pas } \\
\text { significatif }\end{array}$ & Ns & ns & Ns & ns & ns \\
\hline $\begin{array}{l}\text { Entreprises } \\
\text { bénéficiaires }\end{array}$ & oui & non & non & oui & $\begin{array}{l}\text { Le résultat net et le flux } \\
\text { de trésorerie de } \\
\text { financement sont } \\
\text { significatifs }\end{array}$ & 70.7 & 60.0 & 55 & 57 & $\begin{array}{l}\text { Le résultat net } \\
\text { présente le contenu } \\
\text { informationnel le plus } \\
\text { élevé }\end{array}$ \\
\hline $\begin{array}{l}\text { Entreprises } \\
\text { déficitaires }\end{array}$ & non & non & oui & oui & $\begin{array}{l}\text { Les flux de trésorerie de } \\
\text { financement et } \\
\text { d'investissement sont } \\
\text { significatifs }\end{array}$ & 42.5 & 42.0 & 43.4 & 47.9 & $\begin{array}{l}\text { Le flux de trésorerie } \\
\text { de financement } \\
\text { présente le contenu } \\
\text { informationnel le plus } \\
\text { élevé }\end{array}$ \\
\hline $\begin{array}{l}\text { Entreprises } \\
\text { internet }\end{array}$ & oui & non & non & oui & $\begin{array}{l}\text { Le flux de trésorerie de } \\
\text { financement est } \\
\text { significatif. }\end{array}$ & 37.7 & 39.8 & 37.3 & 37.4 & $\begin{array}{l}\text { Le résultat net } \\
\text { présente le contenu } \\
\text { informationnel le plus } \\
\text { élevé }\end{array}$ \\
\hline $\begin{array}{l}\text { Entreprises } \\
\text { technologies de } \\
\text { l'information }\end{array}$ & oui & oui & non & oui & $\begin{array}{l}\text { Seul le coefficient } \\
\text { attaché au flux de } \\
\text { trésorerie } \\
\text { d'investissement n'est } \\
\text { pas significatif }\end{array}$ & 55.6 & 57.0 & 47.4 & 51.4 & indéterminé \\
\hline $\begin{array}{l}\text { Entreprises } \\
\text { "autres valeurs } \\
\text { de croissance" } \\
\end{array}$ & ns & $\mathrm{ns}$ & $\mathrm{ns}$ & ns & $\begin{array}{l}\text { Le modèle n'est pas } \\
\text { significatif }\end{array}$ & ns & ns & ns & $\mathrm{ns}$ & ns \\
\hline $\begin{array}{l}\text { Entreprises } \\
\text { introduites avant } \\
\text { le } 1 \mathrm{er} \text { janvier } \\
2000\end{array}$ & oui & oui & non & oui & $\begin{array}{l}\text { Seul le coefficient } \\
\text { attaché au flux de } \\
\text { trésorerie } \\
\text { d'investissement n'est } \\
\text { pas significatif }\end{array}$ & 52.9 & 53.9 & 46.2 & 48.3 & $\begin{array}{l}\text { Le flux de trésorerie } \\
\text { d'activité présente le } \\
\text { contenu } \\
\text { informationnel le plus } \\
\text { élevé }\end{array}$ \\
\hline $\begin{array}{l}\text { Entreprises } \\
\text { introduites après } \\
\text { le } 1 \mathrm{er} \text { janvier } \\
2000\end{array}$ & oui & non & non & oui & $\begin{array}{l}\text { Le résultat net et le flux } \\
\text { de trésorerie de } \\
\text { financement sont } \\
\text { significatifs }\end{array}$ & 33.7 & 30.3 & 29.1 & 37.1 & indéterminé \\
\hline
\end{tabular}


Pour continuer à croître et stabiliser la situation du fait des pertes, la recherche de capitaux est devenue primordiale, les investisseurs étant désormais enclins au pessimisme.

On note que le bénéfice explique le mieux la capitalisation pour les entreprises bénéficiaires. Investissement et financement, en revanche, importent davantage pour les entreprises déficitaires. La valeur est étroitement associée au résultat et à la capacité de lever des fonds dans le secteur internet auxquels s'ajoute l'aptitude à dégager des liquidités pour les technologies de l'information. Enfin les flux d'activité ont le contenu informationnel le plus élevé pour les entreprises les plus anciennes alors que le flux de financement est prépondérant pour les entreprises nouvellement cotées.

A ce stade, on peut conclure que les mesures comptables, en particulier le résultat net, les flux de trésorerie d'activité et de financement expliquent en grande partie la capitalisation boursière (H1) et que le résultat net de préférence aux flux de trésorerie dispose du pouvoir d'information le plus important $(\mathrm{H} 2)$.

Les flux de trésorerie sont plus ou moins significatifs selon les éléments contingents :

- pour les flux d'activité (introduction en bourse avant 2000 et entreprises de technologies) ;

- pour les flux d'investissement (année 2000 et entreprises déficitaires);

- pour ceux de financement (année 2000, 2001, entreprises bénéficiaires, déficitaires, introduction en bourse avant et après 2000, entreprises internet et de technologies de l'information).

Les flux de trésorerie de financement dominent dans l'explication de la capitalisation quelle que soit la métrique de contingence, contrairement à ce que nous envisagions en H3.

L'analyse selon les différentes métriques montre que les flux de trésorerie d'activité présentent une supériorité informationnelle pour les entreprises introduites avant 2000 et les flux de 
financement externes pour les entreprises déficitaires. Dans tous les autres cas, c'est le résultat net qui véhicule l'information la plus importante.

Cela semble confirmer les travaux de Dechow (1994) sur la supériorité informationnelle du résultat net sur les flux de trésorerie d'activité, qui avait établi ces conclusions, en courte période, lorsque les engagements pèsent sur le résultat et pour les entreprises à court cycle d'exploitation ce qui présente une analogie avec nos données de recherche.

En revanche, nos résultats montrant que tous les indicateurs sauf les flux d'investissement sont significativement associés à la valeur de marché s'opposent à l'étude de Black (1998) sur l'importance du flux d'investissement au cours des premières années du cycle de vie des firmes.

\subsection{Résultat du modèle d'Ohlson}

In fine, le troisième modèle inspiré des travaux d'Ohlson est utilisé en complément des études précédentes pour vérifier s'il améliore les deux premiers modèles. Les premiers résultats (modèle 1 et modèle 2) ont mis en évidence une relation positive entre capitalisation boursière et résultat net. Cependant ce dernier n'est peut être pas le meilleur indicateur de performance pour ces sociétés souvent non rentables.

Pour estimer les opportunités de croissance futures, trois autres variables sont testées :

- le montant du chiffre d'affaires. A priori, il devrait être positivement associé à la valeur de marché, un bon niveau d'activité étant le signe de bénéfices futurs ;

- l'excédent brut d'exploitation qui mesure si l'entreprise est rentable sur le plan opérationnel. Il devrait être positivement associé à la capitalisation ;

- la valeur ajoutée puisqu'elle représente une mesure de la richesse créée par l'entreprise et qu'elle est souvent utilisée pour estimer les opportunités de croissance future. Elle devrait être significativement associée à la capitalisation boursière.

Les mesures de flux sont aussi introduites dans le modèle. 
Le modèle testé est le suivant :

\section{Equation de régression 3 (modèle d'Ohlson):}

$\mathrm{CB}_{\mathrm{i}, \mathrm{t}}=\mathrm{a}_{0}+\mathrm{a}_{1} \mathrm{FP}_{\mathrm{i}, \mathrm{t}}+\mathrm{a}_{2} \mathrm{CA}_{\mathrm{i}, \mathrm{t}}+\mathrm{a}_{3} \mathrm{VA}_{\mathrm{i}, \mathrm{t}}+\mathrm{a}_{4} \mathrm{EBE}_{\mathrm{i}}+\mathrm{a}_{5} \mathrm{Flux}_{\mathrm{i}, \mathrm{t}}+\mathrm{Eit}$

CB i, t : Capitalisation boursière de l'entreprise i à l'instant $\mathrm{t}$;

Fpit : Valeur comptable des fonds propres;

CA : Chiffre d'affaires;

$\mathrm{EBE}^{\mathrm{i}}$ : Résultat d'exploitation brut (avant impôts et taxes, intérêts et des dotations aux amortissement et provisions) de l'entreprise $\mathrm{i}$ à l'instant $\mathrm{t}$;

VA : Valeur ajoutée de l'entreprise i à l'instant t

Flux i,t : Indicateur de flux de trésorerie de l'entreprise i à l'instant $t$ : FTA, FTI, FTF

Ei,t: les termes d'erreurs

\subsubsection{Résultats d'ensemble}

Ils sont présentés dans le tableau 5 ci-après.

Tableau 5: Résultat des régressions entre capitalisation boursière et fondamentaux d'entreprise (modèle 3)

\begin{tabular}{|c|c|c|c|c|c|c|c|c|c|c|c|c|}
\hline & & & & & & & & & & $R^{2}$ & Fisher & D W \\
N=245 & Variables & C & FP & CA & VA & EBE & FTA & FTI & FTF & & & \\
\hline 1. & Coeff & 4155,19 & $\mathbf{2 , 9 0 8} *$ & 0,010 & $-0,447$ & $\mathbf{5 , 1 7 2} *$ & $\mathbf{1 , 7 6 1} *$ & & & $54,19 \%$ & $53,95^{*}$ & 1,788 \\
\hline 2. & Coeff & 5316.136 & $\mathbf{2 . 7 8 2}$ & -0.159 & $-0,316$ & $\mathbf{6 , 4 7 2} *$ & & -0.15 & & $52.5 \%$ & $50,46^{*}$ & 1,84 \\
\hline 3 & Coeff & 9972.34 & $\mathbf{1 . 9 5}^{*}$ & -0.11 & -0.16 & $\mathbf{6 . 3 6 0}^{*}$ & & & $\mathbf{1 . 2 7}^{*}$ & $55.6 \%$ & $57.32^{*}$ & 1.9 \\
\hline
\end{tabular}

La qualité d'ajustement est satisfaisante $\left(\mathrm{R}^{2}>50 \%\right)$. Les trois modèles sont significatifs. Les coefficients des fonds propres, de l'excédent brut d'exploitation, des flux de trésorerie d'activité et de financement sont significatifs au seuil de 5\%. Les coefficients du chiffre d'affaires, de la valeur ajoutée et les flux de trésorerie d'investissement ne le sont pas. On les exclut donc comme mesures des opportunités de croissance. Apparemment tout se passe comme si le marché retenait de préférence l'indicateur de résultat et l'indicateur de flux de trésorerie d'activité et de financement, à l'indicateur d'activité, quand celui- ci est disponible.

Tableau 6 : Test de Wald pour le modèle général

\begin{tabular}{|l|l|l|l|}
\hline & Modèle 1 & Modèle 1 & Modèle 3 \\
\hline $\begin{array}{l}\text { Test de l'égalité des } \\
\text { coefficients }\end{array}$ & EBE= FTO & EBE=FTI & EBE=FTF \\
\hline Chi-deux stat & $5.537^{*}$ & $44.5^{*}$ & $25.31^{*}$ \\
\hline P value & $\mathbf{0 . 0 1 8}$ & $\mathbf{0 . 0 0}$ & $\mathbf{0 . 0 0}$ \\
\hline
\end{tabular}


La statistique de Wald (tableau 6) montre que l'EBE et les flux de trésorerie apportent une information différente. Le tableau 7 indique que l'EBE donne la meilleure qualité d'ajustement $\left(\mathrm{R}^{2}=52.5 \%\right)$. Le résultat opérationnel ou économique brut est donc la variable apportant le plus d'information pour expliquer les valeurs de marché des entreprises de la nouvelle économie.

Tableau 7 : Contenu informationnel le plus élevé

\begin{tabular}{|c|c|c|c|c|}
\hline & Modèle EBE & Modèle FTA & Modèle FTI & Modèle FTF \\
\hline cste & 4680.13 & -10185.09 & -12261.63 & -10946.29 \\
\hline $\mathrm{FP}$ & $2.84 *$ & $2.824 *$ & $2.287 *$ & $1.676^{*}$ \\
\hline $\mathrm{CA}$ & -0.171 & $0.312 *$ & 0.142 & 0.144 \\
\hline VA & -0.297 & 0.435 & $1.062 *$ & $1.371 *$ \\
\hline EBE & $6.562 *$ & & & \\
\hline FTA & & 3.071* & & \\
\hline FTI & & & $-0.88 * *$ & \\
\hline FTF & & & & $1.384 *$ \\
\hline $\mathbf{R}^{2}$ & 52.5 & 42.5 & 43.9 & 46.8 \\
\hline
\end{tabular}

Ainsi, le «résultat brut d'exploitation » mesuré par l'EBE c'est-à-dire la capacité à créer de la liquidité d'exploitation et les flux de trésorerie d'activité et de financement (FTA et FTF) sont positivement associés à la valeur de marché.

\subsubsection{Meilleur modèle}

Pour obtenir la meilleure explication des capitalisations boursières, le modèle suivant introduit toutes les variables de flux de trésorerie, d'activité, d'investissement et de financement. En raison de la très forte corrélation entre flux de trésorerie d'investissement et flux de financement, le flux de financement est seul retenu.

Le modèle proposé est :

$\mathrm{CB}_{\mathrm{i}, t}=\mathrm{a}_{0}+\mathrm{a}_{1} \mathrm{FP}_{\mathrm{i}, \mathrm{t}}+\mathrm{a}_{2} \mathrm{CA}_{\mathrm{i}, \mathrm{t}}+\mathrm{a}_{3} \mathrm{VA}_{\mathrm{i}, \mathrm{t}}+\mathrm{a}_{4} \mathrm{EBE}_{\mathrm{i}}+\mathrm{a}_{5} \mathrm{FTA}_{\mathrm{i}, \mathrm{t}}+\mathrm{a}_{6} \mathrm{FTFit}+\varepsilon i \mathrm{it}$

Tableau 8 : Résultat des régressions, capitalisation boursière et mesures comptables (meilleur modèle, période entière).

\begin{tabular}{|c|c|c|c|c|c|c|c|c|c|c|}
\hline Variables & C & $\mathrm{FP}$ & CA & VA & EBE & FTA & FTF & $\mathrm{R}^{2}$ & Fisher & $\begin{array}{l}\text { Durbin } \\
\text { Watson }\end{array}$ \\
\hline Coefficient & 11208,79 & 1,697 & 0,207 & $-0,357$ & 4,004* & $2,883 *$ & $1,776^{*}$ & $59,70 \%$ & $56,09 \% *$ & 1,795 \\
\hline
\end{tabular}


Avec un $\mathrm{R}^{2}$ de $59,7 \%$, ce modèle donne pour la période entière le meilleur résultat. Les mesures comptables sont particulièrement significatives pour expliquer les valorisations obtenues par les entreprises de la nouvelle économie française entre 2000 et 2002. Comme précédemment, a été testé l'effet des différents facteurs de contingence : la conjoncture (année), la performance (résultat net positif ou négatif), le business model (l'appartenance sectorielle) et enfin l'âge (entreprise introduite avant ou après 2000).

En définitive, l'introduction du modèle d'Ohlson complète et améliore les résultats obtenus.

Les hypothèses $\mathrm{H} 1$ et $\mathrm{H} 4$ concernant respectivement la valeur informationnelle des mesures comptables et les variables complémentaires se trouvent confirmées pour H1 puisque les indicateurs testés comme les fonds propres mais tout particulièrement l'excédent brut d'exploitation et les flux de trésorerie issus de l'activité et des opérations financières expliquent en grande partie la capitalisation boursière. En revanche, pour H4 seul l'excédent brut d'exploitation fournit de l'information sur les opportunités de croissance et non le chiffre d'affaires et la valeur ajoutée ${ }^{12}$. Eprouvant ensuite le pouvoir informationnel de l'excédent brut d'exploitation (H5), il apparaît que celui-ci montre le pouvoir d'information le plus élevé.

Quant au pouvoir explicatif du modèle testé, la régression de type Ohlson permet de passer d'une explication de la valeur boursière de $49,5 \%$ à 55,6\%. De plus, la recherche du «meilleur modèle » en intégrant en plus les deux mesures de flux de trésorerie d'activité et de financement l'augmente encore à 59,7\%.

Finalement l'utilisation de la régression de type Ohlson incorporant les deux flux précédents permet d'accroître encore l'explication de la capitalisation boursière quels que soient les facteurs de contingence notamment l'an $2000\left(\mathrm{R}^{2}=84 \%\right)$ ce qui est considérable pour ce type d'entreprises jugées extrêmement difficiles à évaluer, pour les entreprises de technologies de l'information $\left(\mathrm{R}^{2}=79 \%\right)$ et pour les entreprises bénéficiaires $\left(\mathrm{R}^{2}=70 \%\right)$. 


\section{Conclusion générale}

Ces résultats montrent que contrairement à l'idée répandue au cours de la phase haussière récente du cycle boursier, les investisseurs ont probablement donné de l'importance aux mesures comptables des entreprises françaises de la nouvelle économie même si ces sociétés toutes jeunes et aux produits inhabituels pouvaient sembler difficiles à évaluer.

Les mesures comptables ont un sens. La combinaison fonds propres, chiffre d'affaires, résultat net et flux de trésorerie notamment ceux d'activité et de financement explique plus de 50\% de la capitalisation boursière des entreprises. Résultat net, flux de trésorerie d'activité et de financement sont à privilégier en situation d'évaluation. On retiendra que les flux de trésorerie d'investissement ne sont significatifs que l'année 2000 et pour les seules entreprises déficitaires. Le résultat net présente le contenu informationnel le plus élevé et plus généralement l'excédent brut d'exploitation en tant que mesure du résultat opérationnel. Les analystes y sont sensibles voyant là une capacité potentielle de production de liquidité d'exploitation et de financement ce qui concorde avec les travaux de Dechow (1994). La valeur d'une entreprise est celle de ses flux de trésorerie futurs actualisés. Même s'ils sont par définition inconnus des analystes, leur approche indirecte par les résultats publiés constitue une approximation acceptable. Si le marché anticipe ou constate une amélioration, les investisseurs achètent. Dans le cas contraire, ils vendent. On notera aussi que le modèle d'Ohlson améliore systématiquement la compréhension de la valeur de marché de ces firmes. Mais si le pouvoir explicatif des modèles testés est satisfaisant avec une valeur du coefficient de détermination entre $50 \%$ et $60 \%$, sauf en 2000 année pour laquelle la qualité d'ajustement du modèle est la meilleure et atteint $70 \%$ puis $84 \%$, d'autres informations ont aussi leur place pour comprendre l'écart de valeur inexpliqué. C'est le cas pour des éléments physiques relatifs à l'information publiée ou mal représentés par la comptabilité comme les différentes formes de capital humain ou structurel, la présence et la 
création d'actifs immatériels, les variables stratégiques ou tout simplement l'application de méthodes comptables encore en évolution et à améliorer. 
1.Fama et French (1995) montrent que les titres à fort ratio «market to book » présentent des rendements élevés car ils rémunèrent des risques plus importants comme le risque de faillite.

2.Biddle, Seow et Siegel (1995) ont montré que les bénéfices sont pertinents même si dans certains secteurs les flux de trésorerie d'activité peuvent l'être. Les auteurs examinent les variables suivantes: flux de trésorerie d'activité, chiffre d'affaires et bénéfices.

3. «The primary deficiency of the existing (valuation) literature is that too little thought has been given to what economic message could be conveyed by a given disclosure, and how that message may vary across situations»..... «It would be interesting to study how fundamental analysis for new firms differ from that for established firms». Bernard (1989).

4.L'étude porte sur un échantillon de 37961 observations (entreprises - années) sur la période 1976-1995. La méthodologie utilisée s'inspire des travaux d'Anthony et Ramesh (1992) qui classent et notent les entreprises par cycle de vie en fonction de trois indicateurs : le taux de distribution des dividendes, la croissance du chiffre d'affaires (ventes) et enfin l'âge de l'entreprise. Six groupes sont définis : «start up »(172 observations), croissance (7162 observations), croissance -maturité ( 7350 observations), maturité (6169 observations) et maturité déclin (8514observations) et déclin (8754 observations).

5.L'indice ITCAC, créé par euronext en mars 2000, est un indice technologique transversal qui comprend l'ensemble des valeurs technologiques françaises Il intègre plus de 150 valeurs issues de tous les segments de marché de la cote des actions.

6.La base de données Osiris a été retenue en raison de la présence de deux entreprises étrangères dans l'échantillon. Les rapports annuels proviennent du site de l'AMF.

7.La nomenclature sectorielle FTSE est appliquée pour détailler la composition de l'échantillon. 8.Cash from operations : flux de trésorerie d'activité, cash from investing activities: flux de trésorerie utilisés par l'investissement, cash from financing activities : flux de trésorerie apportés par les activités de financement ; résultat net comptable.

9.Selon la méthodologie proposée par Biddle, Seow et Siegel (1995) et Black (1998). Biddle, Seow et Siegel (1995) étudient le contenu informationnel du résultat net et du flux de trésorerie d'exploitation selon le secteur d'activité des entreprises, et Black (1998) selon le cycle de vie de l'entreprise.

10.Le test de Wald permet d'identifier si des variables présentent un contenu informationnel différent. Ce test met en évidence le fait que deux variables fournissent une information différente sans pour autant identifier celle qui a le pouvoir explicatif le plus important.

11.Durbin Watson : test de l'auto-corrélation des erreurs

12.Sauf pour les entreprises en déficit. 


\section{Bibliographie}

AMIR, E. et LEV, B. (1996), «Value-relevance of non financial information: The wireless communication industry », Journal of Accounting and Economics, vol. 22, n¹, pp. 33-21.

ANTHONY, J.H et RAMESH, K. (1992), "Association between Accounting Performance Measures and Stock Prices : A Test of the Life Cycle Hypothesis», Journal of Accounting and Economics, vol. 15, pp. 203-227.

BARTH, M.E., BEAVER, W.H. et LANDSMAN, W.R. (1996), «Valuation Characteristics of Equity Book Value and Net Income: Test of Abandonment Option Hypothesis», Working paper, Stanford University.

BERNARD, V. (1989), «Capital Markets Research in the Accounting during the 1980's : A Critical Review», The state of accounting research as we enter the 1990's, Thomas Frecka editor, University of Illinois, pp. 72-120.

BERNARD, V. (1994), «Accounting based Valuation Methods, Determinants of Market to Book Ratios and Implications of Financial Analysis», Working paper, University of Michigan.

BIDDLE, G.C., SEOW, G.S. et SIEGEL, A. (1995), «Relative versus Incremental Information Content», Contemporary Accounting Research, vol.12, n ${ }^{\circ}$, pp. 1-23.

BLACK, E.L. (1998), «Life Cycle Impacts on the incremental value relevance of earnings and cash flow measures» Journal of Financial Statement Analysis, vol. 4, pp. 40-56.

BURGSTALHER, D.C. et DICHEV, I.D.(1997), «Earnings, Adaptation and Equity value», The Accounting Review, vol. 72, pp. 187-215.

CAVANAUGH, J.K et GAREN, J. (1997), «Assets Specificity, Unionization and the Firm's use of Debt», Managerial and Decision Economics, vol. 18, n³, pp. 255-270.

CAZAVAN-JENY, A. (2004), «Le ratio market to book et la reconnaissance des immatériels une étude du marché français », Comptabilité-Contrôle-Audit, vol. 10, n², pp. 99-124.

DEMERS, E. et LEV, B. (2000), «A Rude Awakening: Internet value drivers in 2000», Review of Accounting Studies, vol.6, n²-3, pp. 331-359.

DECHOW, P. (1994), «Accountings Earnings and Cash Flows as Measure of Firm Performance: The Role of Accounting Accruals», Journal of Accounting and Economics, vol.18, pp. 3-42.

DING, Y. et STOLOWY, H.(2003), «Les facteurs déterminants de la stratégie des groupes français en matière de communication sur les activités de $\mathrm{R} \& \mathrm{D}$ », Finance Contrôle Stratégie, vol.6, n 1 , pp. 39-62.

FAMA, E.F. et FRENCH, K.F.(1995), «Size and Book-to-Market Factors in Earnings and Returns», Journal of Finance, vol.50, $\mathrm{n}^{\circ} 1$, pp. 131-156.

FRANKEL, R. et LEE, C.(1998), «Accounting Valuation, Market Expectation, and crosssectional Stock Returns », Journal of Accounting and Economics, vol. 25, n³, pp. 283-319.

HAND, J. (2003), «Profits, Losses, and the Non Linear Pricing of Internet Stocks», in Intangibles Assets : Values, Measures and Risks », eds.J.R.M. Hand and B. Lev, Oxford University Press.

KOTHARI, S., SHANKEN, J. et SLOAN, R. (1995), «Another Look at the Cross-Section of Expected Returns», Journal of Finance, vol. 50, n ${ }^{\circ}$, pp. 185-224. 
LEV, B. et SOUGIANNIS, T. (1996), «The capitalization, amortization and value-relevance of R\&D», Journal of Accounting and Economics, vol. 21, n¹, pp. 107-139.

MYERS, S. (1977), «Determinants of corporate borrowing», Journal of Financial Economics, vol. 5, pp. 147-175.

OHLSON, J. (1995), «Earnings, Book Values and Dividends in Security Valuation », Contemporary Accounting Research, vol. 11, pp. 161-182.

RAJGOPAL, S., KOTHA, S. et VENKATACHALAM, M. (2000a), «The relevance of web traffic for internet stock prices», Working Paper, University of Washington.

RAJGOPAL, S., KOTHA, S. et VENKATACHALAM, M., (2003), «The Value Relevance of Network Advantages : The Case of e-commerce Firms», Journal of Accounting Research, vol. 41, n 1 , pp. 135-162.

RAYBURN, F.R. (1986), «Corporate Financial Accounting», Issues in Accounting Education, vol. $11, \mathrm{n}^{\circ} 1$, pp. $232-234$.

SLOAN, R.G. (1996), «Do Stock Prices Fully Reflect Information in Accruals and Cash Flows About Future Earnings?», Accounting Review, vol. 71, n³, pp. 289-316.

TRUEMAN, B., WONG, M.H. F. et. ZHANG, X (2001), « Back to basics : Forecasting the revenues of Internet firms», Review of Accounting Studies, vol.6, pp. 305-329.

TRUEMAN, B., WONG, M.H. F. et. ZHANG, X. (2002), «The Eyeballs Have It: Searching for the Value in Internet Stocks», Journal of Accounting Research, vol. 38, pp. 137-162. 
Annexe : Statistiques descriptives de l'échantillon : période 2000-2002 ( milliers d'euros))

\begin{tabular}{|l|r|r|r|r|r|r|}
\hline Variables de structure & $\begin{array}{l}\text { Nombre } \\
\text { d'obs. }\end{array}$ & $\begin{array}{l}\text { Moyenne } \\
\text { période }\end{array}$ & $\begin{array}{l}\text { Médiane } \\
\text { période }\end{array}$ & Ecart type & \multicolumn{1}{l}{ Minimum } & Maximum \\
\hline Capitalisation boursière & 245 & 76238,42 & 28095,87 & 138602,24 & 1560,00 & 1344228,6 \\
\hline Fonds propres & 245 & 27064,69 & 15670,05 & 32582,02 & -16752 & 190619 \\
\hline Résultat net & 245 & $-1492,81$ & -365 & 10967,27 & -73503 & 30338 \\
\hline Total actifs & 245 & 61037,08 & 30749 & 83591,33 & 1422 & 579395 \\
\hline Actifs d'exploitation & 245 & 39743,14 & 23496,5 & 57517,42 & 1164 & 475267 \\
\hline Dettes d'exploitation & 245 & 25072,43 & 12133 & 43206,237 & 621 & 336422 \\
\hline Total des produits & 245 & 53438,96 & 28548 & 90928,38 & 566 & 1001306 \\
\hline Chiffre d'affaires & 245 & 51920,67 & 27653,34 & 90642,86 & 625 & 1001306 \\
\hline Marge brute & 245 & 43,92 & 62,48 & 66,552 & $-490,65$ & 99,98 \\
\hline Valeur ajoutée & 245 & 17113,36 & 11359 & 23405,59 & -55815 & 142013 \\
\hline EBEE & 245 & 1590,35 & 894,57 & 9983,11 & 1590,35822 & 894,57 \\
\hline Coûts des B\&S vendus & 245 & 28171,782 & 10226 & 69685,79 & 0,76 & 816076 \\
\hline Effectifs & 245 & 438 & 272 & 652,3 & 24 & 6257 \\
\hline Flux de trésorerie d'activité & 245 & $-4015,24$ & $-1468,5$ & 17002,41 & -164804 & 36568 \\
\hline Flux de trésorerie d'investissement & 245 & $-5920,72$ & -945 & 23496,647 & -175222 & 95795 \\
\hline Flux de trésorerie de financement & 245 & 10451,16 & 2437,5 & 30214,246 & -115968 & 223529 \\
\hline
\end{tabular}


